of the larger component. Because of the slight depth of eclipse and the trouble with comparison stars, the above results by themselves cannot be considered as anything more than suggestive. However, E. F. Carpenter's observations taken in the blue, yellow, and ultra-violet on this night and the preceding one, show this effect very clearly and leave little doubt of its reality.

It should further be noted that if the present fragmentary results prove to be a fair sample, the system is free from those erratic light changes which add such complexities to the interpretation of other systems of this sort.

Flower and Cook Observatories, University of Pennsylvania.

\section{Woolard, Edgar W. A comparison of Brown's Lunar Tables with the theory from which they were constructed.}

For 60 dates at half-day intervals, from 1948 April 24.0 to May 24.0 UT, the longitude and latitude of the moon to two decimals of a second of arc and the parallax to three decimals were taken from Brown's tables and compared with values that had been computed to 5 decimals directly from Brown's theoretical expressions by the Selective Sequence Electronic Computer of International Business Machines Corporation.

Significant differences between the SSEC and the tabular values were evident in the longitude and in the latitude. The discrepancy in the longitude is very small but is systematic, the principal part apparently having a period of about a month, with an amplitude of the order of $\mathbf{O}$. I $\mathbf{I}$; the discrepancy in the latitude is strongly periodic, with an amplitude about o". I5 and a period about a month.

An analysis of these differences to determine their source appeared advisable. The SSEC computations were therefore compared in detail with the tabular computations for the longitude on 14 selected dates, and for the latitude on 12 of these dates. The differences are for the most part satisfactorily accounted for by approximations and expedients adopted by Brown and Hedrick in the construction of the tables to facilitate their practical use, and are within the standards of accuracy that were set for the tables. The large discrepancy in the latitude, however, is principally due to an oversight in the tables; in constructing the tables, the effect of the long period variations of the lunar inclination upon several of the large terms in the latitude was inadvertently included twice.
The resulting error in the tabular latitude is large enough to be detected in observations; it has been found in a comparison of the tabular latitude with the observed latitude obtained with the 6-inch transit circle at the U. S. Naval Observatory during 1929-r949.

\section{U. S. Naval Observatory, \\ Washington, D. C}

Wouthuysen, S. A. On the excitation mechanism of the $2 \mathrm{I}-\mathrm{cm}$ (radio-frequency) interstellar hydrogen emission line.

The mechanism proposed here is a radiative one: as a consequence of absorption and re-emission of Lyman- $\alpha$ resonance radiation, a redistribution over the two hyperfine-structure components of the ground level will take place. Under the assumption-here certainly permitted - that induced emissions can be negelcted, it can easily be shown that the relative distribution of the two levels in question, under stationary conditions, will depend solely on the shape of the radiation spectrum in the $\mathrm{L} \alpha$ region, and not on the absolute intensity.

The shape of the spectrum of resonance radiation, quasi-imprisoned in a large gas cloud, could only be determined by a careful study of the "scattering" process (absorption and re-emission) in a cloud of definite shape and dimensions. The spectrum will turn out to depend upon the localization in the cloud.

Some features can be inferred from more general considerations. Take a gas in a large container, with perfectly reflecting walls. Let the gas be in equilibrium at temperature $T$, together with Planck radiation of that same temperature. The scattering processes will not affect the radiation spectrum. One can infer from this fact that the photons, after an infinite number of scattering processes on gas atoms with kinetic temperature $T$, will obtain a statistical distribution over the spectrum proportional to the Planck-radiation spectrum of temperature $T$. After a finite but large number of scattering processes the Planck shape will be produced in a region around the initial frequency.

Photons reaching a point far inside an interstellar gas cloud, with a frequency near the $\mathrm{L} \alpha$ resonance frequency, will have suffered on the average a tremendous number of collisions. Hence in that region, which is wider the larger the optical depth of the cloud is for the Lyman radiation, the Planck spectrum corresponding to the gas-kinetic temperature will be established 
as far as the shape is concerned. Because, however, the relative occupation of the two hyperfine-structure components of the ground state depends only upon the shape of the spectrum near the $L \alpha$ frequency, this occupation will be the one corresponding to equilibrium at the gas temperature.

The conclusion is that the resonance radiation provides a long-range interaction between gas atoms, which forces the internal (spin-)degree of freedom into thermal equilibrium with the thermal motion of the atoms.

\section{Institute for Theoretical Physics of the City University, Amsterdam.}

\section{Zechiel, Leon N. and Geoffrey Keller. A survey of eclipsing binary systems showing apsidal motion.}

Thirty eclipsing binary systems of known or suspected apsidal motion were analyzed to determine whether a correlation could be made between the mass distribution within the stars and the spectral type. A set of combined photometric and spectroscopic elements for each system was assembled. Some systems have not been observed spectroscopically, and the values of the eccentricity and the apsidal period had to be estimated from photometric data alone in these cases. The data has been tabulated for all systems which have been adequately observed. Fourteen cases in which apsidal motion has been indicated, but for which the data are insufficient to support detailed analysis, were rejected.

The final sets of elements for each system were analyzed by the method of Sterne, yielding the apsidal coefficients, $k_{2}$, which are a measure of the degree of central condensation of the mass of the stars. Values of the effective polytropic index of each star were obtained from the quantities $k_{2}$ in the usual manner. The absolute dimensions of the systems were derived from the elements by various methods suited to the data available in each case.

The final results were embodied in a table, and a plot of the effective polytropic index versus the spectral type was made. A similar plot was constructed from the analysis by Russell in 1939 . A comparison shows considerable change in the plot due to the reclassification of the spectra of several of the stars and to the inclusion of new data. There appears to be a limitation of $n_{\text {ef } f}$ to values between 2.9 and 4 . I, with the lower values tending to be associated with earlier spectral types. The ratio of central density to mean density is 54 for a polytrope of index 3.0 and $6 \mathrm{I} 4$ for a polytrope of index 4.o. While the stars in this survey were not assumed to be polytropes these two cases represent models having values of $k_{2}$ corresponding roughly to the observed range. The spectral types represented in the survey ranged from $\mathrm{O} 8.5$ to $\mathrm{F} 2$.

Perkins Observatory, Delaware, Ohio.

TITLES OF ADDITIONAL PAPERS PRESENTED AT THE MEETING IN CLEVELAND, OHIO

Anderson, J. Pamelia. The position of the moon at the time of the I 948 eclipse.

Bidelman, W. P. and W. W. Morgan. A remarkable O-type star.

Binnendijk, L. The space distribution of interstellar material in the Milky Way.

Bok, Bart J. and Margaret Olmsted. Magnitude standards for the southern hemisphere.

Cook, Allan F. II. Radiative equilibrium in a hydrogen atmosphere.

Eckert, W. J., Rebecca B. Jones and H. K. Clark. A precise lunar ephemeris.

Genatt, Sol H. Note on a graphical method for the prediction of occultations.

Goldberg, Leo, R. R. McMath, O. C. Mohler and A. K. Pierce. Identification of $C O$ in the solar atmosphere.

Harwood, Margaret. The nova-like variable CM Aquilae.

Henriksen, S. W. Note on the kinematics of the moon's motion.

Johnson, Harold L. Magnitude systems.

McKellar, Andrew, G. J. Odgers and L. H. Aller. The chromospheric K-line during the recent eclipse of $3 \mathrm{I}$ Cygni.

Mears, D. D. Field techniques for occultation observation. Millis, John. The genesis of Saturn and its rings.

Neyman, J. and C. D. Shane. A model of spatial distribution of galaxies. Preliminary report.

O'Keefe, John A. and J. Pamelia Anderson. Calculation of the earth's radius from occultation data.

Osterbrock, Donald A. The time of relaxation for stars in a fluctuating density field.

Panay, T. N. and John A. O'keefe. Progress on the measurements of darkening at the sun's limb from the results of the 1948 eclipse.

Scott, Elizabeth R. Theoretical counterparts of certain observable distributions relating to galaxies.

Swope, Henrietta H. Photographic magnitudes and colors in the globular cluster NGC 6397.

Thomsen, Warren J. The path and orbit of the detonating meteor of August 29, I95I.

White, Marvin S. Note on the accuracy of Hayn's charts as measured by photoelectric observation.

Wrubel, Marshal $\mathrm{H}$. On the decay of a primeval stellar magnetic field.

Wylie, C. C. The path and orbit of the detonating meteor of July 28 , I95I. 